\title{
Explicit Scheme for Fixed Point Problem for Nonexpansive Semigroup and Split Equilibrium Problem in Hilbert Space
}

\author{
Pei Zhou and Gou-Jie Zhao \\ School of Management, Tianjin University, Tianjin 300072, China \\ Correspondence should be addressed to Pei Zhou; zhoupei2011@163.com
}

Received 1 May 2014; Revised 18 July 2014; Accepted 1 August 2014; Published 19 August 2014

Academic Editor: Filomena Cianciaruso

Copyright ( 2014 P. Zhou and G.-J. Zhao. This is an open access article distributed under the Creative Commons Attribution License, which permits unrestricted use, distribution, and reproduction in any medium, provided the original work is properly cited.

We establish an iterative method for finding a common element of the set of fixed points of nonexpansive semigroup and the set of split equilibrium problems. Under suitable conditions, some strong convergence theorems are proved. Our works improve previous results for nonexpansive semigroup.

\section{Introduction}

Let $H$ be a real Hilbert space whose inner product and norm are denoted by $\langle\cdot, \cdot\rangle$ and $\|\cdot\|$, respectively. Let $C$ be a nonempty closed convex subset of $H$, and let $F$ be a bifunction of $C \times$ $C$ into $\mathbb{R}$ which is the set of real numbers. The equilibrium problem introduced by Blum and Oettli [1] for $F: C \times C \rightarrow$ $\mathbb{R}$ is to find $x \in C$ such that

$$
F(x, y) \geq 0, \quad \forall y \in C .
$$

The set of solutions of (1) is denoted by $\operatorname{EP}(F)$. Numerous problems in physics, optimization, and economics reduce to finding a solution of (1) (see [2-8]). The split equilibrium problem was introduced by Moudafi in [9]; he considers the following pair of equilibrium problems in different spaces: let $H_{1}$ and $H_{2}$ be two real Hilbert spaces, let $F_{1}: C \times C \rightarrow R$ and $F_{2}: Q \times Q \rightarrow R$ be nonlinear bifunctions, and let $A: H_{1} \rightarrow H_{2}$ be a bounded linear operator, and consider the nonempty closed convex subsets $C \subseteq H_{1}$ and $Q \subseteq H_{2}$; then the split equilibrium problem (SEP) is to find $x^{*} \in C$ such that

$$
F_{1}\left(x^{*}, x\right) \geq 0, \quad \forall x \in C,
$$

and such that

$$
y^{*} \in A x^{*} \in Q, \quad F_{2}\left(y^{*}, y\right) \geq 0, \quad \forall y \in Q .
$$

The solution set of SEP (2)-(3) is denoted by $\Omega=\{p \in$ $\left.\mathrm{EP}\left(F_{1}\right): A p \in \operatorname{EP}\left(F_{2}\right)\right\}$.

Recall that mapping $T$ of $C$ into itself is called nonexpansive if

$$
\|T x-T y\| \leq\|x-y\|, \quad \forall x, y \in C .
$$

Let a family $S=(T(s))_{s \geq 0}$ be a nonexpansive semigroup on $H$, if it satisfies the following conditions:

(S1) $T(0) x=x \forall x \in H$;

(S2) $T(s+t)=T(s) T(t)$ for each $s, t \geq 0$;

(S3) $\|T(s) x-T(t) y\| \leq\|x-y\|$ for each $x, y \in H$ and $s \geq 0$;

(S4) $\forall x \in H, s \rightarrow T(s) x$ is continuous.

The set of all the common fixed points of family $S$ is denoted by $F_{i x}(S)$; that is,

$$
F_{i x}(S)=\{x \in C: T(s) x=x, 0 \leq s<\infty\}=\bigcap_{0 \leq s<\infty} F_{i x}(T(s)),
$$

where $F_{i x}(T(s))$ is the set of fixed points of $T(s)$. It is well known that $F_{i x}(S)$ is closed and convex.

In 2010, Tian [10] introduced the following general iterative scheme for finding an element of set of solutions to the fixed point of nonexpansive mapping in a Hilbert space. Define the sequence $\left\{x_{n}\right\}$ by

$$
x_{n+1}=\alpha_{n} \gamma f\left(x_{n}\right)+\left(1-\mu \alpha_{n} B\right) T x_{n},
$$


where $B$ is $k$-Lipschitzian and $\eta$-strongly monotone operator. Then he proved that if the sequence $\left\{\alpha_{n}\right\}$ satisfies appropriate conditions, the sequence $\left\{x_{n}\right\}$ generated by (6) converges strongly to the unique solution $x^{*} \in F_{i x}(T)$ of the variational inequality

$$
\left\langle(\gamma f-\mu B) x^{*}, x-x^{*}\right\rangle \leq 0, \quad \forall x \in F_{i x}(T) .
$$

In 2011, Ceng et al. [11] added the metric project to the method of Tian (6) and studied the following explicit iterative scheme to find fixed points:

$$
x_{n+1}=P_{C}\left[\alpha_{n} \gamma f\left(x_{n}\right)+\left(1-\mu \alpha_{n} B\right) T x_{n}\right]
$$

They prove the strong convergence of $x_{n}$ to a fixed point $x^{*} \in$ $F_{i x}(T)$ of the same variational inequality (7).

In 2008, Plubtieng and Punpaeng [12] introduced the following implicit iterative algorithm to prove a strong convergence theorem for fixed point problem with nonexpansive semigroup:

$$
x_{n}=\alpha_{n} f\left(x_{n}\right)+\left(1-\alpha_{n}\right) \frac{1}{s_{n}} \int_{0}^{s_{n}} T(s) x_{n} d s,
$$

where $x_{n}$ is a continuous net and $s_{n}$ is a positive real divergent net.

In 2014, Kazmi and Rizvi [13] studied the following implicit iterative algorithm. Under some assumptions, they obtain some strong convergence theorem for EP (1) and the fixed point problem:

$$
\begin{gathered}
u_{n}=T_{r_{n}}^{F_{1}}\left(x_{n}+\delta A^{*}\left(T_{r_{n}}^{F_{2}}-I\right) A x_{n}\right), \\
x_{n}=\alpha_{n} \gamma f\left(x_{n}\right)+\left(I-\alpha_{n} B\right) \frac{1}{s_{n}} \int_{0}^{s_{n}} T(s) u_{n} d s,
\end{gathered}
$$

where $s_{n}$ and $r_{n}$ are the continuous nets in $(0,1)$.

Motivated and inspired by [10-13], we introduce an explicit iterative scheme for finding a common element of the set of solutions SEP and fixed point for a nonexpansive semigroup in real Hilbert spaces. Starting with an arbitrary $x_{1} \in H$, define sequences $\left\{x_{n}\right\}$ and $\left\{u_{n}\right\}$ by

$$
\begin{gathered}
u_{n}=T_{r_{n}}^{F_{1}}\left(x_{n}+\delta A^{*}\left(T_{r_{n}}^{F_{2}}-I\right) A x_{n}\right), \\
x_{n+1}=P_{C}\left[\alpha_{n} \gamma f\left(x_{n}\right)+\left(I-\mu \alpha_{n} B\right) \frac{1}{s_{n}} \int_{0}^{s_{n}} T(s) u_{n} d s\right] .
\end{gathered}
$$

Under suitable conditions, some strong convergence theorems for approximating to these common elements are proved.

\section{Preliminaries}

This section collects some results that will be used in the proofs.

Let $H$ be a real Hilbert space with the inner product $\langle\cdot, \cdot\rangle$ and the norm $\|\cdot\|$, respectively.
It is well known that, for all $x, y \in H$ and $\lambda \in[0,1]$, the following holds:

$$
\begin{aligned}
\|\lambda x+(1-\lambda) y\|^{2}= & \lambda\|x\|^{2}+(1-\lambda)\|y\|^{2} \\
& -\lambda(1-\lambda)\|x-y\|^{2} .
\end{aligned}
$$

Let $C$ be a nonempty closed convex subset of $H$. Then, for any $x \in H$, there exists a unique nearest point of $C$, denoted by $P_{C} x$, such that $\left\|x-P_{C} x\right\| \leq\|x-y\|$ for all $y \in C$. Such $P_{C}$ is called the metric projection from $H$ into $C$. We know that $P_{C}$ is nonexpansive. It is also known that $P_{C} x \in C$ and

$$
\left\langle x-P_{C} x, P_{C} x-z\right\rangle \geq 0, \quad \forall x \in H, z \in C .
$$

It is easy to see that (13) is equivalent to

$$
\|x-z\|^{2} \geq\left\|x-P_{C} x\right\|^{2}+\left\|P_{C} x-z\right\|^{2}, \quad \forall x \in H, z \in C .
$$

Let $B: C \rightarrow H$ be a nonlinear mapping. Recall the following definitions.

Definition 1. $B$ is said to be

(i) monotone if

$$
\langle B x-B y, x-y\rangle \geq 0, \quad \forall x, y \in C,
$$

(ii) strongly monotone if there exists a constant $\alpha>0$ such that

$$
\langle B x-B y, x-y\rangle \geq \alpha\|x-y\|^{2}, \quad \forall x, y \in C,
$$

for such a case, $B$ is said to be $\alpha$-strongly monotone,

(iii) $\alpha$-inverse strongly monotone $(\alpha$-ism) if there exists a constant $\alpha>0$ such that

$$
\langle B x-B y, x-y\rangle \geq \alpha\|B x-B y\|^{2}, \quad \forall x, y \in C,
$$

(iv) $k$-Lipschitz continuous if there exists a constant $k \geq 0$ such that

$$
\|B x-B y\| \leq k\|x-y\|, \quad \forall x, y \in C .
$$

Remark 2. Let $\mathscr{F}=\mu B-\gamma f$, where $B$ is a $k$-Lipschitz and $\eta$-strongly monotone operator on $H$ with $k>0$ and $f$ is a Lipschitz mapping on $H$ with coefficient $L>0,0<\gamma \leq \mu \eta / L$. It is a simple matter to see that the operator $\mathscr{F}$ is $(\mu \eta-\gamma L)$ strongly monotone over $H$; that is,

$$
\begin{array}{r}
\langle\mathscr{F} x-\mathscr{F} y, x-y\rangle \geq(\mu \eta-\gamma L)\|x-y\|^{2}, \\
\forall(x, y) \in H \times H .
\end{array}
$$

Lemma 3 (see [14]). Let $T$ be a nonexpansive mapping of a closed convex subset $C$ of a Hilbert space $H$. If $T$ has a fixed point, then $I-T$ is demiclosed; that is, whenever the sequence of $x_{n}$ is weakly convergent to $x$ and $(I-T) x_{n}$ is strongly convergent to $y$, then $(I-T) x=y$. 
Lemma 4 (see [15]). Let $C$ be a nonempty bounded closed convex subset of a Hilbert space $H$ and let $(T(s))_{s \geq 0}$ be a nonexpansive semigroup on $C$. Then, for each $h \geq 0$,

$$
\lim _{t \rightarrow+\infty} \sup _{x \in C}\left\|\frac{1}{t} \int_{0}^{t} T(s) x d s-T(h) \frac{1}{t} \int_{0}^{t} T(s) x d s\right\|=0 .
$$

Definition 5 (see [9]). A mapping $T: H \rightarrow H$ is said to be averaged if it can be written as the average of the identity mapping and a nonexpansive mapping; that is,

$$
T=(1-\varepsilon) I+\varepsilon S,
$$

where $\varepsilon \in(0,1), S: H \rightarrow H$ is nonexpansive, and $I$ is the identity operator on $H$.

Proposition 6 (see [9]).

(i) If $T=(1-\varepsilon) S+\varepsilon V$, where $S: H \rightarrow H$ is averaged, $V: H \rightarrow H$ is nonexpansive, and $\varepsilon \in(0,1)$, then $T$ is averaged.

(ii) The composite of finitely many averaged mappings is averaged.

(iii) If $T$ is $\nu$-ism, then, for $\gamma>0, \gamma T$ is $(\nu / \gamma)$-ism.

(iv) $T$ is averaged if, and only if, its complement $I-T$ is $v$-ism for some $v>1 / 2$.

Assumption 7 (see [1]). For solving the equilibrium problem for a bifunction $F: C \times C \rightarrow \mathbb{R}$, let us assume that $F$ satisfies the following conditions:

(A1) $F(x, x)=0$ for all $x \in C$;

(A2) $F$ is monotone, that is, $F(x, y)+F(y, x) \leq 0$ for all $x, y \in C$;

(A3) for each $x, y, z \in C$,

$$
\lim _{t \rightarrow 0} F(t z+(1-t) x, y) \leq F(x, y)
$$

(A4) for each $x \in C, y \mapsto F(x, y)$ is convex and lower semicontinuous.

Lemma 8 (see [2]). Let $C$ be a nonempty closed convex subset of $H$, and let $F$ be a bifunction of $C \times C$ into $\mathbb{R}$ satisfying (A1)(A4). Let $r>0$ and $x \in H$. Then there exists $z \in C$ such that

$$
F(z, y)+\frac{1}{r}\langle y-z, z-x\rangle \geq 0, \quad \forall y \in C
$$

Define a mapping $T_{r}: H \rightarrow C$ as follows:

$$
T_{r}^{F}(x)=\left\{z \in C: F(z, y)+\frac{1}{r}\langle y-z, z-x\rangle \geq 0, \forall y \in C\right\}
$$

for all $x \in H$. Then the following hold:

(1) $T_{r}^{F}$ is single-valued;
(2) $T_{r}^{F}$ is firmly nonexpansive; that is, for any $x, y \in H$,

$$
\left\|T_{r}^{F} x-T_{r}^{F} y\right\|^{2} \leq\left\langle T_{r} x-T_{r} y, x-y\right\rangle
$$

(3) $F_{i x}\left(T_{r}^{F}\right)=E P(F)$;

(4) $E P(F)$ is closed and convex.

Lemma 9 (see [16]). Let $C$ be a nonempty closed convex subset of a Hilbert space $H$, and let $F: C \times C \rightarrow \mathbb{R}$ be a bifunction. Let $x \in C$ and $r_{1}, r_{2} \in(0, \infty)$. Then

$$
\left\|T_{r_{1}}^{F} x-T_{r_{2}}^{F} x\right\| \leq\left|1-\frac{r_{2}}{r_{1}}\right|\left(\left\|T_{r_{1}}^{F} x\right\|+\|x\|\right) .
$$

Lemma 10 (see [17]). Assume that $\lambda \in(0,1)$ and $\mu>0$. Let $B$ be a $B: H \rightarrow H$ which is $\eta$-strongly monotone and $\theta$ Lipschitzian on $H$ with $\theta>0, \eta>0$. Let $0<\mu<2 \eta / \theta^{2}$. Then the operator $I-\lambda \mu B$ is contraction; that is, $\|I-\lambda \mu B\| \leq(1-\lambda \tau)$, where $\tau=1-\sqrt{1-\mu\left(2 \eta-\mu \theta^{2}\right)} \in(0,1]$.

Lemma 11 (see [10]). Let $H$ be a Hilbert space, $f: H \rightarrow H$ is a contractive mapping with constant $\beta \in(0,1) . B: H \rightarrow H$ is $\theta$-Lipschitzian and $\eta$-strongly monotone operator with $\theta>0$, $\eta>0$. Then, for $0<\sigma<\mu \eta / \beta$,

$$
\begin{array}{r}
\langle x-y,(\mu B-\sigma f) x-(\mu B-\sigma f) y\rangle \geq(\mu \eta-\sigma \beta)\|x-y\|^{2}, \\
x, y \in H .
\end{array}
$$

That is, $\mu B-\sigma f$ is strongly monotone with coefficient $\mu \eta-\sigma \beta$.

Lemma 12. Let $H$ be a real Hilbert space. Then the following well-known results hold: $\forall x, y \in H$,

$$
\|x+y\|^{2} \leq\|x\|^{2}+2\langle y, x+y\rangle .
$$

Lemma 13 (see [18]). Assume that $\left\{\alpha_{n}\right\}$ is a sequence of nonnegative real numbers such that

$$
\alpha_{n+1} \leq\left(1-\gamma_{n}\right) \alpha_{n}+\delta_{n}, \quad n \geq 0,
$$

where $\left\{\alpha_{n}\right\}$ is a sequence in $(0,1)$ and $\left\{\delta_{n}\right\}$ is a sequence in $R$ such that

(i) $\sum_{n=1}^{\infty} \gamma_{n}=\infty$,

(ii) $\lim \sup _{n \rightarrow \infty}\left(\delta_{n} / \gamma_{n}\right) \leq 0$ or $\sum_{n=1}^{\infty}\left|\delta_{n}\right|<\infty$.

Then $\lim _{n \rightarrow \infty} \alpha_{n}=0$.

\section{The General Explicit Iterative Method}

Let $f: H_{1} \rightarrow H_{1}$ be a contractive mapping with constant $\beta \in(0,1)$ and let $B: H_{1} \rightarrow H_{1}$ be $\eta$-strongly monotone and $\theta$-Lipschitzian with $\theta>0, \eta>0$. Let $0<\mu<2 \eta / \theta^{2}$ and $0<\gamma<\mu\left(\eta-\mu \theta^{2} / 2\right) / \beta=\tau / \beta$. Let $S=\{T(s): 0 \leq s<\infty\}$ be a nonexpansive semigroup on $C$ such that $\Gamma=F_{i x}(S) \bigcap \Omega \neq \emptyset$. Assume $\left\{r_{n}\right\}$ and $\left\{s_{n}\right\}$ are the continuous nets of positive real numbers such that $\lim _{n \rightarrow 0} r_{n}=r>0$ and $\lim _{n \rightarrow 0} s_{n}=+\infty$. 
In this section, we introduce the following explicit iterative scheme that the nets $\left\{u_{n}\right\}$ and $\left\{x_{n}\right\}$ are generated by

$$
\begin{gathered}
u_{n}=T_{r_{n}}^{F_{1}}\left(x_{n}+\delta A^{*}\left(T_{r_{n}}^{F_{2}}-I\right) A x_{n}\right), \\
x_{n+1}=P_{C}\left[\alpha_{n} \gamma f\left(x_{n}\right)+\left(I-\mu \alpha_{n} B\right) \frac{1}{s_{n}} \int_{0}^{s_{n}} T(s) u_{n} d s\right],
\end{gathered}
$$

where $\delta \in(0,1 / L), L$ is the spectral radius of the operator $A^{*} A$, and $A^{*}$ is the adjoint of $A$.

We prove the strong convergence of $\left\{u_{n}\right\}$ and $\left\{x_{n}\right\}$ to a fixed point $x^{*}$ of $S$ which solves the following variational inequality:

$$
\left\langle(\mu F-\gamma f) x^{*}, x^{*}-\bar{x}\right\rangle \leq 0, \quad \forall \bar{x} \in \Gamma=F_{i x}(S) \bigcap \Omega .
$$

In the sequel, we denote by $y_{n}$ the sequence defined by

$$
y_{n}=\frac{1}{s_{n}} \int_{0}^{s_{n}} T(s) u_{n} d s .
$$

Next, we will prove first a lemma and then some corollaries to be used in the proofs for the main result of this section.

Theorem 14. Let $H_{1}$ and $H_{2}$ be two real Hilbert spaces and let $C \subseteq H_{1}$ and $Q \subseteq H_{2}$ be nonempty closed subsets. Let $A: H_{1} \rightarrow H_{2}$ be a bounded linear operator. Assume that $F_{1}: C \times C \rightarrow \mathbb{R}$ and $F_{2}: Q \times Q \rightarrow \mathbb{R}$ are the bifunctions satisfying Assumption 7 and $F_{2}$ is upper semicontinuous in the first argument. Let the sequences $\left\{u_{n}\right\}$ and $\left\{x_{n}\right\}$ be generated by (30), and suppose that the sequence $\left\{\alpha_{n}\right\}$ satisfies the following conditions:

(a) $\alpha_{n} \in(0,1)$ and $\lim _{n \rightarrow \infty} \alpha_{n}=0$;

(b) $\sum_{n=0}^{\infty} \alpha_{n}=0$;

(c) either $\sum_{n=0}^{\infty}\left|\alpha_{n+1}-\alpha_{n}\right|<\infty$ or $\lim _{n \Rightarrow \infty}\left(\alpha_{n} / \alpha_{n+1}\right)=1$.

Then the sequences $\left\{u_{n}\right\}$ and $\left\{x_{n}\right\}$ converge strongly to $x^{*} \in$ $\Gamma=F_{i x}(S) \bigcap \Omega$, where $x^{*}=P_{\Gamma}(I-\mu B+\gamma f) x^{*}$, which is the unique solution of the variational inequality (31).

Proof. We divide the proof into several steps.

(i) $\left\{x_{n}\right\}$ is well defined. $\mathrm{H}_{2}$ by

For $\alpha_{n} \in(0,1)$ and $\forall x \in H_{1}$, define a mapping $G: H_{1} \rightarrow$

$$
\begin{aligned}
G x=P_{C}[ & \alpha_{n} \gamma f(x)+\left(I-\mu \alpha_{n} B\right) \frac{1}{s_{n}} \\
& \left.\times \int_{0}^{s_{n}} T(s) T_{r_{n}}^{F_{1}}\left(x+\delta A^{*}\left(T_{r_{n}}^{F_{2}}-I\right) A x\right) d s\right] .
\end{aligned}
$$

According to Lemma 8, we can easily know that $T_{r_{1}}^{F_{1}}$ and $T_{r_{2}}^{F_{2}}$ both are firmly nonexpansive mappings and are averaged operators. From Proposition 6, we can obtain that the operator $\left(I+\delta A^{*}\left(T_{r_{n}}^{F_{2}}-I\right) A\right)$ is averaged and hence nonexpansive. Following Lemma 9 and $\forall x, y \in H_{1}$, we get

$$
\begin{aligned}
& \|G x-G y\| \\
& =\| P_{C}\left[\alpha_{n} \gamma f+\left(I-\mu \alpha_{n} B\right) \frac{1}{s_{n}}\right. \\
& \left.\times \int_{0}^{s_{n}} T(s) T_{r_{n}}^{F_{1}}\left(I+\delta A^{*}\left(T_{r_{n}}^{F_{2}}-I\right) A\right) d s\right] x \\
& -P_{C}\left[\alpha_{n} \gamma f+\left(I-\mu \alpha_{n} B\right) \frac{1}{s_{n}}\right. \\
& \left.\times \int_{0}^{s_{n}} T(s) T_{r_{n}}^{F_{1}}\left(I+\delta A^{*}\left(T_{r_{n}}^{F_{2}}-I\right) A\right) d s\right] y \| \\
& \leq \|\left[\alpha_{n} \gamma f+\left(I-\mu \alpha_{n} B\right) \frac{1}{s_{n}}\right. \\
& \left.\times \int_{0}^{s_{n}} T(s) T_{r_{n}}^{F_{1}}\left(I+\delta A^{*}\left(T_{r_{n}}^{F_{2}}-I\right) A\right) d s\right] x \\
& -\left[\alpha_{n} \gamma f+\left(I-\mu \alpha_{n} B\right) \frac{1}{s_{n}}\right. \\
& \left.\times \int_{0}^{s_{n}} T(s) T_{r_{n}}^{F_{1}}\left(I+\delta A^{*}\left(T_{r_{n}}^{F_{2}}-I\right) A\right) d s\right] y \| \\
& \leq \alpha_{n} \gamma\|f(x)-f(y)\|+\left(1-\alpha_{n} \tau\right) \\
& \times \| \frac{1}{s_{n}} \int_{0}^{s_{n}} T(s)\left[T_{r_{n}}^{F_{1}}\left(x+\delta A^{*}\left(T_{r_{n}}^{F_{2}}-I\right) A x\right) d s\right. \\
& \left.-T_{r_{n}}^{F_{1}}\left(y+\delta A^{*}\left(T_{r_{n}}^{F_{2}}-I\right) A y\right) d s\right] \| \\
& \leq \alpha_{n} \gamma \beta\|x-y\|+\left(1-\alpha_{n} \tau\right) \\
& \times \| T_{r_{n}}^{F_{1}}\left(x+\delta A^{*}\left(T_{r_{n}}^{F_{2}}-I\right) A x\right) d s \\
& -T_{r_{n}}^{F_{1}}\left(y+\delta A^{*}\left(T_{r_{n}}^{F_{2}}-I\right) A y\right) d s \| \\
& \leq \alpha_{n} \gamma \beta\|x-y\|+\left(1-\alpha_{n} \tau\right)\|x-y\| \\
& =\left(1-\alpha_{n}\left(\tau-\alpha_{n} \beta\right)\right)\|x-y\| \text {. }
\end{aligned}
$$

Since $0<1-\alpha_{n}\left(\tau-\alpha_{n} \beta\right)<1$, we can claim that the mapping $G$ is a contraction mapping. Therefore, by Banach contraction principle, $G$ has the unique fixed point $x^{*}$.

(ii) The sequences $\left\{u_{n}\right\},\left\{y_{n}\right\}$, and $\left\{x_{n}\right\}$ are bounded.

Letting $p \in \Gamma=F_{i x}(S) \bigcap \Omega$, we obtain that $p=T_{r_{n}}^{F_{1}} p$, $A p=T_{r_{n}}^{F_{2}} A p$, and $p=T(s) p$. 
From (30), we obtain

$$
\begin{aligned}
& \left\|u_{n}-p\right\|^{2} \\
& =\left\|T_{r_{n}}^{F_{1}}\left(I+\delta A^{*}\left(T_{r_{n}}^{F_{2}}-I\right) A\right) x_{n}-p\right\|^{2} \\
& =\left\|T_{r_{n}}^{F_{1}}\left(I+\delta A^{*}\left(T_{r_{n}}^{F_{2}}-I\right) A\right) x_{n}-T_{r_{n}}^{F_{1}} p\right\|^{2} \\
& \leq\left\|x_{n}+\delta A^{*}\left(T_{r_{n}}^{F_{2}}-I\right) A x_{n}-p\right\|^{2} \\
& \leq\left\|x_{n}-p\right\|^{2}+\left\|\delta A^{*}\left(T_{r_{n}}^{F_{2}}-I\right) A x_{n}\right\|^{2} \\
& +2 \delta\left\langle x_{n}-p, A^{*}\left(T_{r_{n}}^{F_{2}}-I\right) A x_{n}\right\rangle \\
& \leq\left\|x_{n}-p\right\|^{2}+\delta^{2}\left\langle\left(T_{r_{n}}^{F_{2}}-I\right) A x_{n}, A A^{*}\left(T_{r_{n}}^{F_{2}}-I\right) A x_{n}\right\rangle \\
& +2 \delta\left\langle A\left(x_{n}-p\right),\left(T_{r_{n}}^{F_{2}}-I\right) A x_{n}\right\rangle \\
& \leq\left\|x_{n}-p\right\|^{2}+L \delta^{2}\left\langle\left(T_{r_{n}}^{F_{2}}-I\right) A x_{n},\left(T_{r_{n}}^{F_{2}}-I\right) A x_{n}\right\rangle \\
& +2 \delta\left\langle A\left(x_{n}-p\right)+\left(T_{r_{n}}^{F_{2}}-I\right) A x_{n}\right. \\
& \left.-\left(T_{r_{n}}^{F_{2}}-I\right) A x_{n}, A^{*}\left(T_{r_{n}}^{F_{2}}-I\right) A x_{n}\right\rangle \\
& \leq\left\|x_{n}-p\right\|^{2}+L \delta^{2}\left\|\left(T_{r_{n}}^{F_{2}}-I\right) A x_{n}\right\|^{2} \\
& +2 \delta\left\{\left\langle T_{r_{n}}^{F_{2}} A x_{n}-A p,\left(T_{r_{n}}^{F_{2}}-I\right) A x_{n}\right\rangle\right. \\
& \left.-\left\|\left(T_{r_{n}}^{F_{2}}-I\right) A x_{n}\right\|^{2}\right\} \\
& \leq\left\|x_{n}-p\right\|^{2}+L \delta^{2}\left\|\left(T_{r_{n}}^{F_{2}}-I\right) A x_{n}\right\|^{2} \\
& +2 \delta\left\{\frac{1}{2}\left\|\left(T_{r_{n}}^{F_{2}}-I\right) A x_{n}\right\|^{2}-\left\|\left(T_{r_{n}}^{F_{2}}-I\right) A x_{n}\right\|^{2}\right\} \\
& \leq\left\|x_{n}-p\right\|^{2}+L \delta^{2}\left\|\left(T_{r_{n}}^{F_{2}}-I\right) A x_{n}\right\|^{2}-\delta\left\|\left(T_{r_{n}}^{F_{2}}-I\right) A x_{n}\right\|^{2} \\
& =\left\|x_{n}-p\right\|^{2}+\delta(L \delta-1)\left\|\left(T_{r_{n}}^{F_{2}}-I\right) A x_{n}\right\|^{2} .
\end{aligned}
$$

Since $\delta \in(0,1 / L)$, we have

$$
\left\|u_{n}-p\right\| \leq\left\|x_{n}-p\right\| .
$$

From (32), we have

$$
\begin{aligned}
\left\|y_{n}-p\right\| & =\left\|\frac{1}{s_{n}} \int_{0}^{s_{n}} T(s) u_{n} d s-p\right\| \\
& \leq \frac{1}{s_{n}}\left\|\int_{0}^{s_{n}}\left(T(s) u_{n}-T(s) p\right) d s\right\| \\
& \leq\left\|u_{n}-p\right\| \leq\left\|x_{n}-p\right\| .
\end{aligned}
$$

Further, using (31) again, we obtain

$$
\begin{aligned}
\left\|x_{n}-p\right\| & \left\|P_{C}\left[\alpha_{n} \gamma f\left(x_{n}\right)+\left(I-\mu \alpha_{n} B\right) \frac{1}{s_{n}} \int_{0}^{s_{n}} T(s) u_{n} d s\right]-p\right\| \\
\leq & \left\|\alpha_{n} \gamma f\left(x_{n}\right)+\left(I-\mu \alpha_{n} B\right) \frac{1}{s_{n}} \int_{0}^{s_{n}} T(s) u_{n} d s-p\right\| \\
= & \| \alpha_{n}\left(\gamma f\left(x_{n}\right)-\mu B p\right)+\left(I-\mu \alpha_{n} B\right) \frac{1}{s_{n}} \\
& \times \int_{0}^{s_{n}} T(s) u_{n} d s-\left(I-\mu \alpha_{n} B\right) p \| \\
\leq & \alpha_{n}\left\|\gamma f\left(x_{n}\right)-\mu B p\right\|+\left(1-\alpha_{n} \tau\right) \\
& \times\left\|\frac{1}{s_{n}} \int_{0}^{s_{n}} T(s) u_{n} d s-p\right\| \\
\leq & \alpha_{n}\left\|\gamma f\left(x_{n}\right)-\mu B p\right\|+\left(1-\alpha_{n} \tau\right) \frac{1}{s_{n}} \\
& \times \int_{0}^{s_{n}}\left\|T(s) u_{n}-T(s) p\right\| d s \\
\leq & \alpha_{n} \gamma\left\|f\left(x_{n}\right)-f(p)\right\|+\alpha_{n}\|\gamma f(p)-\mu B p\| \\
& +\left(1-\alpha_{n} \tau\right)\left\|u_{n}-p\right\| \\
= & {\left[1-\alpha_{n}(\tau-\gamma \beta)\right]\left\|u_{n}-p\right\|+\alpha_{n}\|\gamma f(p)-\mu B p\| . }
\end{aligned}
$$

It follows from (40) and induction that

$$
\left\|x_{n}-p\right\| \leq \frac{\|\gamma f(p)-\mu B p\|}{\tau-\gamma \beta} .
$$

Hence, the sequence $\left\{x_{n}\right\}$ is bounded and therefore $\left\{u_{n}\right\},\left\{y_{n}\right\}$, and $\left\{f\left(x_{n}\right)\right\}$ are also bounded.

(iii) Consider that $\lim _{n \Rightarrow \infty}\left\|x_{n}-u_{n}\right\|=0$.

According to (35) and Lemma 12, we obtain

$$
\left\|x_{n}-p\right\|^{2}
$$

$$
\begin{aligned}
\leq & \left(1-\alpha_{n} \tau\right)^{2}\left\|\frac{1}{s_{n}} \int_{0}^{s_{n}} T(s) u_{n} d s-p\right\|^{2} \\
& +2 \alpha_{n}\left\langle\gamma f\left(x_{n}\right)-\gamma f(p)+\gamma f(p)-\mu B p, x_{n}-p\right\rangle \\
\leq & \left(1+\alpha_{n}^{2} \tau^{2}\right)\left\|u_{n}-p\right\|^{2}+2 \alpha_{n} \gamma \beta\left\|x_{n}-p\right\|^{2} \\
& +2 \alpha_{n}\left\langle\gamma f(p)-\mu B p, x_{n}-p\right\rangle
\end{aligned}
$$




$$
\begin{aligned}
\leq & \left\|u_{n}-p\right\|^{2}+\alpha_{n} \tau^{2}\left\|x_{n}-p\right\|^{2}+2 \alpha_{n} \gamma \beta\left\|x_{n}-p\right\|^{2} \\
& +2 \alpha_{n}\|\gamma f(p)-\mu B p\|\left\|x_{n}-p\right\| \\
\leq & \left\|x_{n}-p\right\|^{2}+\delta(L \delta-1)\left\|\left(T_{r_{n}}^{F_{2}}-I\right) A x_{n}\right\|^{2} \\
& +\alpha_{n} \tau^{2}\left\|x_{n}-p\right\|^{2}+2 \alpha_{n} \gamma \beta\left\|x_{n}-p\right\|^{2} \\
& +2 \alpha_{n}\|\gamma f(p)-\mu B p\|\left\|x_{n}-p\right\| .
\end{aligned}
$$

From (40), we obtain

$$
\begin{aligned}
\delta(1-L \delta) & \left\|\left(T_{r_{n}}^{F_{2}}-I\right) A x_{n}\right\|^{2} \\
\leq \alpha_{n}( & \left(\tau^{2}+2 \gamma \beta\right)\left\|x_{n}-p\right\|^{2} \\
& \left.+2\|\gamma f(p)-\mu B p\|\left\|x_{n}-p\right\|\right) .
\end{aligned}
$$

Since $x_{n}$ is bounded, $\lim _{n \rightarrow \infty} \alpha_{n}=0$, and $\delta(1-L \delta)>0$, we obtain that

$$
\lim _{n \rightarrow \infty}\left\|\left(T_{r_{n}}^{F_{2}}-I\right) A x_{n}\right\|^{2}=0 .
$$

From (30), we have

$$
\begin{aligned}
& \left\|u_{n}-p\right\|^{2} \\
& =\left\|T_{r_{n}}^{F_{1}}\left(I+\delta A^{*}\left(T_{r_{n}}^{F_{2}}-I\right) A\right) x_{n}-p\right\|^{2} \\
& =\left\|T_{r_{n}}^{F_{1}}\left(I+\delta A^{*}\left(T_{r_{n}}^{F_{2}}-I\right) A\right) x_{n}-T_{r_{n}}^{F_{1}} p\right\|^{2} \\
& \leq\left\langle u_{n}-p, x_{n}+\delta A^{*}\left(T_{r_{n}}^{F_{2}}-I\right) A x_{n}-p\right\rangle \\
& =\frac{1}{2}\left\{\left\|u_{n}-p\right\|^{2}+\left\|x_{n}+\delta A^{*}\left(T_{r_{n}}^{F_{2}}-I\right) A x_{n}-p\right\|^{2}\right. \\
& \left.\quad-\left\|u_{n}-p-\left[x_{n}+\delta A^{*}\left(T_{r_{n}}^{F_{2}}-I\right) A x_{n}-p\right]\right\|^{2}\right\} \\
& \leq \frac{1}{2}\left\{\left\|u_{n}-p\right\|^{2}+\left\|x_{n}-p\right\|^{2}\right. \\
& \left.\quad-\left\|u_{n}-x_{n}-\delta A^{*}\left(T_{r_{n}}^{F_{2}}-I\right) A x_{n}\right\|^{2}\right\} \\
& \leq \frac{1}{2}\left\{\left\|u_{n}-p\right\|^{2}+\left\|x_{n}-p\right\|^{2}-\left\|u_{n}-x_{n}\right\|^{2}\right. \\
& \quad-\delta^{2}\left\|A^{*}\left(T_{r_{n}}^{F_{2}}-I\right) A x_{n}\right\|^{2} \\
& \left.\quad+2 \delta\left\|A\left(u_{n}-x_{n}\right)\right\|\left\|\left(T_{r_{n}}^{F_{2}}-I\right) A x_{n}\right\|\right\} .
\end{aligned}
$$

Hence, we obtain

$$
\begin{aligned}
& \left\|u_{n}-p\right\|^{2} \\
& \leq\left\|x_{n}-p\right\|^{2}-\left\|u_{n}-x_{n}\right\|^{2}-\delta^{2}\left\|A^{*}\left(T_{r_{n}}^{F_{2}}-I\right) A x_{n}\right\|^{2} \\
& \quad+2 \delta\left\|A\left(u_{n}-x_{n}\right)\right\|\left\|\left(T_{r_{n}}^{F_{2}}-I\right) A x_{n}\right\|
\end{aligned}
$$

$$
\begin{aligned}
\leq & \left\|x_{n}-p\right\|^{2}-\left\|u_{n}-x_{n}\right\|^{2} \\
& +2 \delta\left\|A\left(u_{n}-x_{n}\right)\right\|\left\|\left(T_{r_{n}}^{F_{2}}-I\right) A x_{n}\right\| .
\end{aligned}
$$

It follows from (40) and (44) that

$$
\begin{aligned}
\| x_{n}- & p \|^{2} \\
\leq & \left\|u_{n}-p\right\|^{2}+\alpha_{n} \tau^{2}\left\|x_{n}-p\right\|^{2}+2 \alpha_{n} \gamma \beta\left\|x_{n}-p\right\|^{2} \\
& +2 \alpha_{n}\|\gamma f(p)-\mu B p\|\left\|x_{n}-p\right\| \\
\leq & \left\|x_{n}-p\right\|^{2}-\left\|u_{n}-x_{n}\right\|^{2} \\
& +2 \delta\left\|A\left(u_{n}-x_{n}\right)\right\|\left\|\left(T_{r_{n}}^{F_{2}}-I\right) A x_{n}\right\| \\
& +\alpha_{n} \tau^{2}\left\|x_{n}-p\right\|^{2}+2 \alpha_{n} \gamma \beta\left\|x_{n}-p\right\|^{2} \\
& +2 \alpha_{n}\|\gamma f(p)-\mu B p\|\left\|x_{n}-p\right\| \\
= & \left\|x_{n}-p\right\|^{2}-\left\|u_{n}-x_{n}\right\|^{2} \\
& +2 \delta\left\|A\left(u_{n}-x_{n}\right)\right\|\left\|\left(T_{r_{n}}^{F_{2}}-I\right) A x_{n}\right\|+\alpha_{n} M_{1},
\end{aligned}
$$

where $M_{1}=\tau^{2}\left\|x_{n}-p\right\|^{2}+2 \gamma \beta\left\|x_{n}-p\right\|^{2}+2 \| \gamma f(p)-$ $\mu B p\|\| x_{n}-p \|$. From (46), we obtain

$$
\left\|u_{n}-x_{n}\right\|^{2} \leq 2 \delta\left\|A\left(u_{n}-x_{n}\right)\right\|\left\|\left(T_{r_{n}}^{F_{2}}-I\right) A x_{n}\right\|+\alpha_{n} M_{1} .
$$

Since $x_{n}$ is bounded, $\lim _{n \rightarrow \infty} \alpha_{n}=0$, and $\delta>0$ and considering (42), we can claim that

$$
\lim _{n \rightarrow \infty}\left\|u_{n}-x_{n}\right\|=0
$$

(iv) Consider that $\lim _{n \rightarrow \infty}\left\|x_{n+1}-x_{n}\right\|=0$.

From (30) and Lemma 13, we have

$$
\begin{aligned}
& \left\|u_{n}-u_{n-1}\right\| \\
& =\| T_{r_{n}}^{F_{1}}\left(x_{n}+\delta A^{*}\left(T_{r_{n}}^{F_{2}}-I\right) A x_{n}\right) \\
& \quad-T_{r_{n-1}}^{F_{1}}\left(x_{n-1}+\delta A^{*}\left(T_{r_{n-1}}^{F_{2}}-I\right) A x_{n-1}\right) \| \\
& \leq \|\left(x_{n}+\delta A^{*}\left(T_{r_{n}}^{F_{2}}-I\right) A x_{n}\right) \\
& \quad-\left(x_{n-1}+\delta A^{*}\left(T_{r_{n}}^{F_{2}}-I\right) A x_{n-1}\right) \| \\
& +\left|1-\frac{r_{n-1}}{r_{n}}\right| \| T_{r_{n}}^{F_{1}}\left(x_{n}+\delta A^{*}\left(T_{r_{n}}^{F_{2}}-I\right) A x_{n}\right) \\
& \quad-\left(x_{n}+\delta A^{*}\left(T_{r_{n}}^{F_{2}}-I\right) A x_{n}\right) \|
\end{aligned}
$$




$$
\begin{aligned}
& \leq\left\|x_{n}-x_{n-1}-\delta A^{*} A\left(x_{n}-x_{n-1}\right)\right\| \\
& +\delta\|A\|\left\|T_{r_{n}}^{F_{2}} A x_{n}-T_{r_{n-1}}^{F_{2}} A x_{n-1}\right\| \\
& +\left|1-\frac{r_{n-1}}{r_{n}}\right| \| T_{r_{n}}^{F_{1}}\left(x_{n}+\delta A^{*}\left(T_{r_{n}}^{F_{2}}-I\right) A x_{n}\right) \\
& -\left(x_{n}+\delta A^{*}\left(T_{r_{n}}^{F_{2}}-I\right) A x_{n}\right) \| \\
& \leq\left(\left\|x_{n}-x_{n-1}\right\|^{2}-2 \delta\left\|A\left(x_{n}-x_{n-1}\right)\right\|^{2}\right. \\
& \left.+\delta^{2}\|A\|^{4}\left\|x_{n}-x_{n-1}\right\|^{2}\right)^{1 / 2} \\
& +\delta\|A\|\left(\left\|A\left(x_{n}-x_{n-1}\right)\right\|\right. \\
& \left.+\left|1-\frac{r_{n-1}}{r_{n}}\right|\left\|T_{r_{n}}^{F_{2}} A x_{n}-A x_{n-1}\right\|\right) \\
& +\left|1-\frac{r_{n-1}}{r_{n}}\right| \| T_{r_{n}}^{F_{1}}\left(x_{n}+\delta A^{*}\left(T_{r_{n}}^{F_{2}}-I\right) A x_{n}\right) \\
& -\left(x_{n}+\delta A^{*}\left(T_{r_{n}}^{F_{2}}-I\right) A x_{n}\right) \| \\
& \leq\left(1-2 \delta\|A\|^{2}+\delta^{2}\|A\|^{4}\right)^{1 / 2}\left\|x_{n}-x_{n-1}\right\| \\
& +\delta\|A\|^{2}\left(\left\|x_{n}-x_{n-1}\right\|\right. \\
& \left.+\left|1-\frac{r_{n-1}}{r_{n}}\right|\left\|T_{r_{n}}^{F_{2}} A x_{n}-A x_{n-1}\right\|\right) \\
& +\left|1-\frac{r_{n-1}}{r_{n}}\right| \| T_{r_{n}}^{F_{1}}\left(x_{n}+\delta A^{*}\left(T_{r_{n}}^{F_{2}}-I\right) A x_{n}\right) \\
& =\left\|x_{n}-x_{n-1}\right\|+\left|1-\frac{r_{n-1}}{r_{n}}\right|\left(\delta\|A\| \varepsilon_{n}+\xi_{n}\right), \\
& \varepsilon_{n}=\left\|T_{r_{n}}^{F_{2}} A x_{n}-A x_{n-1}\right\|, \\
& \xi_{n}=\| T_{r_{n}}^{F_{1}}\left(x_{n}+\delta A^{*}\left(T_{r_{n}}^{F_{2}}-I\right) A x_{n}\right) \\
& -\left(x_{n}+\delta A^{*}\left(T_{r_{n}}^{F_{2}}-I\right) A x_{n}\right) \| . \\
& \left\|y_{n}-y_{n-1}\right\| \\
& =\left\|\frac{1}{s_{n}} \int_{0}^{s_{n}} T(s) u_{n} d s-\frac{1}{s_{n-1}} \int_{0}^{s_{n-1}} T(s) u_{n-1} d s\right\| \\
& \leq\left\|\frac{1}{s_{n}} \int_{0}^{s_{n}} T(s) u_{n} d s-\frac{1}{s_{n}} \int_{0}^{s_{n}} T(s) u_{n-1} d s\right\| \\
& +\left\|\frac{1}{s_{n}} \int_{0}^{s_{n}} T(s) u_{n-1} d s-\frac{1}{s_{n-1}} \int_{0}^{s_{n-1}} T(s) u_{n-1} d s\right\| \\
& \leq \frac{1}{s_{n}} \int_{0}^{s_{n}}\left\|T(s)\left(u_{n}-u_{n-1}\right)\right\| d s \\
& +\left\|\frac{1}{s_{n}} \int_{0}^{s_{n}} T(s) u_{n-1} d s-\frac{1}{s_{n-1}} \int_{0}^{s_{n-1}} T(s) u_{n-1} d s\right\| \\
& \leq\left\|u_{n}-u_{n-1}\right\|+\left|\frac{1}{s_{n}}-\frac{1}{s_{n-1}}\right|\left\|\int_{0}^{s_{n-1}} T(s) u_{n-1} d s\right\| \\
& +\frac{1}{s_{n}}\left\|\int_{s_{n-1}}^{s_{n}} T(s) u_{n-1} d s\right\| .
\end{aligned}
$$$$
-\left(x_{n}+\delta A^{*}\left(T_{r_{n}}^{F_{2}}-I\right) A x_{n}\right) \|
$$$$
\leq\left(1-\delta\|A\|^{2}\right)\left\|x_{n}-x_{n-1}\right\|
$$$$
+\delta\|A\|^{2}\left(\left\|x_{n}-x_{n-1}\right\|+\left|1-\delta\|A\| \frac{r_{n-1}}{r_{n}}\right|\right.
$$$$
\left.\times\left\|T_{r_{n}}^{F_{2}} A x_{n}-A x_{n-1}\right\|\right)
$$$$
+\left|1-\frac{r_{n-1}}{r_{n}}\right| \| T_{r_{n}}^{F_{1}}\left(x_{n}+\delta A^{*}\left(T_{r_{n}}^{F_{2}}-I\right) A x_{n}\right)
$$$$
-\left(x_{n}+\delta A^{*}\left(T_{r_{n}}^{F_{2}}-I\right) A x_{n}\right) \|
$$$$
=\left\|x_{n}-x_{n-1}\right\|+\delta\|A\|\left|1-\frac{r_{n-1}}{r_{n}}\right|
$$$$
\times\left\|T_{r_{n}}^{F_{2}} A x_{n}-A x_{n-1}\right\|
$$$$
+\left|1-\frac{r_{n-1}}{r_{n}}\right| \| T_{r_{n}}^{F_{1}}\left(x_{n}+\delta A^{*}\left(T_{r_{n}}^{F_{2}}-I\right) A x_{n}\right)
$$$$
-\left(x_{n}+\delta A^{*}\left(T_{r_{n}}^{F_{2}}-I\right) A x_{n}\right) \|
$$

Following (48) and (50), we obtain

$$
\begin{aligned}
& \left\|y_{n}-y_{n-1}\right\| \\
& \leq\left(\left\|x_{n}-x_{n-1}\right\|+\left|1-\frac{r_{n-1}}{r_{n}}\right|\left(\delta\|A\| \varepsilon_{n}+\xi_{n}\right)\right) \\
& \quad+\left|\frac{1}{s_{n}}-\frac{1}{s_{n-1}}\right|\left\|\int_{0}^{s_{n-1}} T(s) u_{n-1} d s\right\|+\frac{1}{s_{n}}\left\|\int_{s_{n-1}}^{s_{n}} T(s) u_{n-1} d s\right\| .
\end{aligned}
$$

Using (30) again, we obtain

$$
\begin{aligned}
& \left\|x_{n+1}-x_{n}\right\| \\
& =\| P_{C}\left[\alpha_{n} \gamma f\left(x_{n}\right)+\left(I-\mu \alpha_{n} B\right) y_{n}\right] \\
& \quad-P_{C}\left[\alpha_{n-1} \gamma f\left(x_{n-1}\right)+\left(I-\mu \alpha_{n-1} B\right) y_{n-1}\right] \| \\
& \leq \| \alpha_{n} \gamma f\left(x_{n}\right)+\left(I-\mu \alpha_{n} B\right) y_{n}-\alpha_{n-1} \gamma f\left(x_{n-1}\right) \\
& \quad-\left(I-\mu \alpha_{n-1} B\right) y_{n-1} \| \\
& =\| \alpha_{n} \gamma\left(f\left(x_{n}\right)-f\left(x_{n-1}\right)\right)+\gamma\left(\alpha_{n}-\alpha_{n-1}\right) f\left(x_{n-1}\right) \\
& \quad+\left(I-\mu \alpha_{n} B\right)\left(y_{n}-y_{n-1}\right)+\mu\left(\alpha_{n}-\alpha_{n-1}\right) y_{n-1} \|
\end{aligned}
$$




$$
\begin{aligned}
\leq & \alpha_{n} \gamma \beta\left\|x_{n}-x_{n-1}\right\|+\gamma\left|\alpha_{n}-\alpha_{n-1}\right|\left\|f\left(x_{n-1}\right)\right\| \\
& +\left(1-\alpha_{n} \tau\right)\left\|y_{n}-y_{n-1}\right\|+\mu\left|\alpha_{n}-\alpha_{n-1}\right|\left\|y_{n-1}\right\| \\
\leq & \alpha_{n} \gamma \beta\left\|x_{n}-x_{n-1}\right\|+\gamma\left|\alpha_{n}-\alpha_{n-1}\right|\left\|f\left(x_{n-1}\right)\right\| \\
& +\left(1-\alpha_{n} \tau\right)\left(\left\|x_{n}-x_{n-1}\right\|+\left|1-\frac{r_{n-1}}{r_{n}}\right|\left(\delta\|A\| \varepsilon_{n}+\xi_{n}\right)\right) \\
& +\left|\frac{1}{s_{n}}-\frac{1}{s_{n-1}}\right|\left\|\int_{0}^{s_{n-1}} T(s) u_{n-1} d s\right\| \\
& +\frac{1}{s_{n}}\left\|\int_{s_{n-1}}^{s_{n}}(s) u_{n-1} d s|+\mu| \alpha_{n}-\alpha_{n-1} \mid\right\| y_{n-1} \| \\
= & \left(1-\alpha_{n}(\tau-\gamma \beta)\right) \\
& +\left(\left\|x_{n}-x_{n-1}\right\|+\gamma\left|\alpha_{n}-\alpha_{n-1}\right|\left\|f\left(x_{n-1}\right)\right\|\right. \\
& +M_{2}\left(\gamma\left|\alpha_{n}-\alpha_{n-1}\right|+\left|1-\frac{r_{n-1}}{s_{n}}\right|+\left|\frac{1}{s_{n}}-\frac{1}{s_{n-1}}\right|\right. \\
& +\left|\frac{1}{s_{n}}-\frac{1}{s_{n-1}}\right|\left\|\int_{0}^{s_{n-1}} T(s) u_{n-1} d s\right\|+\mu\left|\alpha_{n}-\alpha_{n-1}\right|\left\|y_{n-1}\right\| \\
& \left.+\left|1-\frac{r_{n-1}}{r_{n}}\right|\left(\delta\|A\| \varepsilon_{n}+\xi_{n}\right)\right)
\end{aligned}
$$

where

$$
\begin{aligned}
& M_{2}=\max \left\{\sup _{n \geq 1}\left(\delta\|A\| \varepsilon_{n}+\xi_{n}\right),\right. \\
&\left.\sup _{n \geq 1}\left(\left\|\int_{s_{n}}^{s_{n-1}} T(s) u_{n-1} d s\right\|\right), \sup _{n \geq 1}\left\|y_{n-1}\right\|\right\} .
\end{aligned}
$$

Since $\left\{x_{n}\right\},\left\{u_{n}\right\}$, and $\left\{y_{n}\right\}$ are bounded, we can claim that $\left\{A x_{n}\right\}$ and $\left\{T(s) u_{n-1}\right\}$ are bounded. We can deduce that $\sup _{n \geq 1}\left(\delta\|A\| \varepsilon_{n}+\xi_{n}\right)<\infty, \sup _{n \geq 1}\left(\left\|\int_{s_{n}}^{s_{n-1}} T(s) u_{n-1} d s\right\|\right)<\infty$ and $\sup _{n \geq 1}\left\|y_{n-1}\right\|<\infty$, and $M_{2}<\infty$.

Following conditions (a)-(c), $\lim _{n \rightarrow 0} r_{n}=r>0$, $\lim _{n \rightarrow 0} s_{n}=+\infty$, and Lemma 13, we obtain that

$$
\lim _{n \rightarrow \infty}\left\|x_{n+1}-x_{n}\right\|=0 .
$$

(v) Consider that $\lim _{n \rightarrow \infty}\left\|T(s) x_{n}-x_{n}\right\|=0$.

$$
\text { From (30) and (32), we obtain }
$$

$$
\begin{aligned}
& \left\|x_{n+1}-y_{n}\right\| \\
& \leq \| P_{C}\left[\alpha_{n} \gamma f\left(x_{n}\right)+\left(I-\mu \alpha_{n} B\right) \frac{1}{s_{n}} \int_{0}^{s_{n}} T(s) u_{n} d s\right] \\
& \quad-P_{C} y_{n} \| \\
& \leq\left\|\alpha_{n} \gamma f\left(x_{n}\right)+\left(I-\mu \alpha_{n} B\right) \frac{1}{s_{n}} \int_{0}^{s_{n}} T(s) u_{n} d s-y_{n}\right\| \\
& \leq \alpha_{n}\left\|\gamma f\left(x_{n}\right)-\mu B \frac{1}{s_{n}} \int_{0}^{s_{n}} T(s) u_{n} d s\right\| .
\end{aligned}
$$

Since $\lim _{n \rightarrow \infty} \alpha_{n}=0$ and $x_{n}$ and $u_{n}$ are bounded, we obtain

$$
\lim _{n \rightarrow \infty}\left\|x_{n+1}-y_{n}\right\|=0
$$

By (54) and (56), we get

$$
\left\|x_{n}-y_{n}\right\| \leq\left\|x_{n}-x_{n+1}\right\|+\left\|x_{n+1}-y_{n}\right\| .
$$

Next, we have

$$
\lim _{n \rightarrow \infty}\left\|x_{n}-y_{n}\right\|=0 .
$$

On the other hand, by (30), we have

$$
\begin{aligned}
& \left\|T(s) x_{n}-x_{n}\right\| \\
& \leq\left\|T(s) x_{n}-T(s) \frac{1}{s_{n}} \int_{0}^{s_{n}} T(s) u_{n} d s\right\| \\
& +\left\|T(s) \frac{1}{s_{n}} \int_{0}^{s_{n}} T(s) u_{n} d s-\frac{1}{s_{n}} \int_{0}^{s_{n}} T(s) u_{n} d s\right\| \\
& \quad+\left\|\frac{1}{s_{n}} \int_{0}^{s_{n}} T(s) u_{n} d s-x_{n}\right\| \\
& \leq\left\|x_{n}-\frac{1}{s_{n}} \int_{0}^{s_{n}} T(s) u_{n} d s\right\| \\
& \quad+\left\|T(s) \frac{1}{s_{n}} \int_{0}^{s_{n}} T(s) u_{n} d s-\frac{1}{s_{n}} \int_{0}^{s_{n}} T(s) u_{n} d s\right\| \\
& \quad+\left\|\frac{1}{s_{n}} \int_{0}^{s_{n}} T(s) u_{n} d s-x_{n}\right\| \\
& \leq 2\left\|x_{n}-y_{n}\right\|+\| T(s) \frac{1}{s_{n}} \int_{0}^{s_{n}} T(s) u_{n} d s \\
& \quad-\frac{1}{s_{n}} \int_{0}^{s_{n}} T(s) u_{n} d s \| .
\end{aligned}
$$

So without loss of generality, we may assume that $S=\{T(s)$ : $0 \leq s<+\infty\}$ is nonexpansive semigroup on $K$, and, by Lemma 4, we obtain

$$
\lim _{n \rightarrow \infty}\left\|T(s) \frac{1}{s_{n}} \int_{0}^{s_{n}} T(s) u_{n} d s-\frac{1}{s_{n}} \int_{0}^{s_{n}} T(s) u_{n} d s\right\| .
$$


From (58), (59), and (60), we have

$$
\lim _{n \rightarrow \infty}\left\|T(s) x_{n}-x_{n}\right\|=0 .
$$

(vi) Consider that $\omega \in \operatorname{EP}\left(F_{1}\right) \bigcap \operatorname{EP}\left(F_{2}\right)$.

Since $\left\{x_{n}\right\}$ is bounded, there exists a subsequence $\left\{x_{n_{i}}\right\}$ of $\left\{x_{n}\right\}$ which converges weakly to $\omega$. From (47), we obtain $\left\{u_{n_{i}}\right\}$ which converges weakly to $\omega$. From (58), $y_{n_{i}} \rightarrow \omega$ follows. We show $\omega \in \operatorname{EP}\left(F_{1}\right)$. According to (30) and (A2),

$$
\frac{1}{r_{n}}\left\langle y-u_{n}, u_{n}-x_{n}\right\rangle \geq F_{1}\left(y, u_{n}\right)
$$

and hence

$$
\left\langle y-u_{n_{i}}, \frac{u_{n_{i}}-x_{n_{i}}}{r_{n_{i}}}\right\rangle \geq F_{1}\left(y, u_{n_{i}}\right) .
$$

Since $\left(u_{n_{i}}-x_{n_{i}}\right) / r_{n_{i}} \rightarrow 0$ and $u_{n_{i}} \rightarrow \omega$, from (A4), it follows that $0 \geq F_{1}(y, \omega)$ for all $y \in H$. For $t$ with $0<t \leq 1$ and $y \in H$, let $y_{t}=t y+(1-t) \omega$; then we get $0 \geq F_{1}\left(y_{t}, \omega\right)$. So, from (A1) and (A4) we have

$$
0=F_{1}\left(y_{t}, y_{t}\right) \leq t F_{1}\left(y_{t}, y\right)+(1-t) F\left(y_{t}, \omega\right) \leq t F_{1}\left(y_{t}, y\right)
$$

and hence $0 \leq F\left(y_{t}, y\right)$. From (A3), we have $0 \leq F_{1}(\omega, y)$ for all $y \in H$. Therefore, $\omega \in \operatorname{EP}\left(F_{1}\right)$.

Since $x_{n_{i}} \rightarrow \omega$ and $A$ is a bounded linear operator, we obtain $A x_{n_{i}} \rightarrow A \omega$. Let $v_{n_{j}}=A x_{n_{j}}-T_{r_{n_{j}}}^{F_{2}} x_{n_{j}}$. Following (42), we obtain $\lim _{n \rightarrow \infty} v_{n_{j}}=0$ and $A x_{n_{j}}-v_{n_{j}}=T_{r_{n_{j}}}^{F_{2}} x_{n_{j}}$. Then from Lemma 8, we get

$$
\begin{gathered}
F_{2}\left(A x_{n_{j}}-v_{n_{j}}, y\right)+\frac{1}{r_{n_{j}}}\left\langle y-\left(A x_{n_{j}}-v_{n_{j}}\right),\left(A x_{n_{j}}-v_{n_{j}}\right)\right. \\
\left.-A x_{n_{j}}\right\rangle \geq 0, \quad \forall y \in Q .
\end{gathered}
$$

Since $F_{2}$ is upper semicontinuous in the first argument, taking limsup to above inequality as $j \rightarrow \infty$ and using $\limsup _{n \rightarrow \infty} r_{n}=r>0$, we obtain that

$$
F_{2}(A \omega, y) \geq 0, \quad \forall y \in Q,
$$

which means that $A \omega \in \operatorname{EP}\left(F_{2}\right)$ and hence $\omega \in \Omega$.

(vii) Consider that $\left\langle(\mu F-\gamma f) x^{*}, x^{*}-\tilde{x}\right\rangle \leq 0, \forall \bar{x} \in \Gamma=$ $F_{i x}(S) \bigcap \Omega$.

According to (30), letting

$$
z_{n}=\alpha_{n} \gamma f\left(x_{n}\right)+\left(I-\mu \alpha_{n} B\right) \frac{1}{s_{n}} \int_{0}^{s_{n}} T(s) u_{n} d s,
$$

we can observe that

$$
\begin{aligned}
x_{n+1}=P_{C} z_{n}= & P_{C} z_{n}-z_{n}+\alpha_{n} \gamma f\left(x_{n}\right) \\
& +\left(I-\mu \alpha_{n} B\right) \frac{1}{s_{n}} \int_{0}^{s_{n}} T(s) u_{n} d s .
\end{aligned}
$$

Observe that

$$
\begin{aligned}
(\mu B-\gamma f) x_{n}= & \frac{1}{\alpha_{n}}\left(P_{C} z_{n}-z_{n}\right)+\frac{1}{\alpha_{n}}\left(x_{n}-x_{n+1}\right) \\
& +\left(I-\mu \alpha_{n} B\right)\left(y_{n}-x_{n}\right) .
\end{aligned}
$$

Hence, for each $p \in \Gamma=F_{i x}(S) \bigcap \Omega$, we can obtain

$$
\begin{aligned}
\left\langle(\mu B-\gamma f) x_{n}, x_{n}-p\right\rangle & \\
= & \frac{1}{\alpha_{n}}\left\langle P_{C} z_{n}-z_{n}, x_{n}-p\right\rangle,+\frac{1}{\alpha_{n}}\left\langle x_{n}-x_{n+1}, x_{n}-p\right\rangle \\
& +\frac{1}{\alpha_{n}}\left\langle\left(I-\mu \alpha_{n} B\right)\left(y_{n}-x_{n}\right), x_{n}-p\right\rangle \\
= & \frac{1}{\alpha_{n}}\left\langle P_{C} z_{n}-z_{n}, x_{n}-p\right\rangle,+\frac{1}{\alpha_{n}}\left\langle x_{n}-x_{n+1}, x_{n}-p\right\rangle \\
& +\frac{1}{\alpha_{n}}\left\langle y_{n}-x_{n}, x_{n}-p\right\rangle-\mu\left\langle B y_{n}-B x_{n}, x_{n}-p\right\rangle .
\end{aligned}
$$

Taking limit $n \rightarrow \infty$ in (70) and noticing that $B y_{n}-B x_{n} \rightarrow$ $B x^{*}-B x^{*}=0, y_{n}-x_{n} \rightarrow \infty$, and $P_{C} z_{n}-z_{n} \rightarrow P_{C} x^{*}-$ $x^{*}=0$, we obtain that

$$
\langle(\mu B-\gamma f) \omega, \omega-p\rangle \leq 0
$$

which implies $\omega=P_{\Gamma}(I-\mu B+\gamma f)$.

(viii) Consider that $\omega \in \Gamma=F_{i x}(S) \bigcap \Omega$. $\Gamma$

From (30), we have $x_{n+1}=P_{C} z_{n}$, and, for any given $x^{*} \in$

$$
\begin{aligned}
x_{n+1}-x^{*}= & P_{C} z_{n}-z_{n}+z_{n}-x^{*} \\
= & P_{C} z_{n}-z_{n}+\alpha_{n}\left(\gamma f\left(x_{n}\right)-\mu B x^{*}\right) \\
& +\left(I-\mu \alpha_{n} B\right) y_{n}-\left(I-\mu \alpha_{n} B\right) x^{*} .
\end{aligned}
$$

Since $P_{C}$ is the metric projection from $H_{1}$ onto $C$, we obtain

$$
\left\langle P_{C} z_{n}-z_{n}, P_{C} z_{n}-x^{*}\right\rangle \leq 0 .
$$

It follows from (72) and (37) that

$$
\begin{aligned}
& \left\|x_{n+1}-x\right\|^{2} \\
& =\left\langle P_{C} z_{n}-z_{n}, x_{n+1}-x^{*}\right\rangle+\alpha_{n}\left\langle\gamma f\left(x_{n}\right)-\mu B x^{*}, x_{n+1}-x^{*}\right\rangle \\
& \quad+\left\langle\left(I-\mu \alpha_{n} B\right)\left(y_{n}-x^{*}\right), x_{n+1}-x^{*}\right\rangle
\end{aligned}
$$




$$
\begin{aligned}
\leq & \alpha_{n}\left\langle\gamma f\left(x_{n}\right)-\mu B x^{*}, x_{n+1}-x^{*}\right\rangle \\
& +\left\langle\left(I-\mu \alpha_{n} B\right)\left(y_{n}-x^{*}\right), x_{n+1}-x^{*}\right\rangle \\
\leq & \alpha_{n} \gamma\left\langle f\left(x_{n}\right)-f\left(x^{*}\right), x_{n+1}-x^{*}\right\rangle \\
& +\alpha_{n}\left\langle\gamma f\left(x^{*}\right)-\mu B x^{*}, x_{n+1}-x^{*}\right\rangle \\
& +\left\langle\left(I-\mu \alpha_{n} B\right)\left(y_{n}-x^{*}\right), x_{n+1}-x^{*}\right\rangle \\
\leq & \alpha_{n} \gamma \beta\left\|x_{n}-x^{*}\right\|\left\|x_{n+1}-x^{*}\right\| \\
& +\alpha_{n}\left\langle\gamma f\left(x^{*}\right)-\mu B x^{*}, x_{n+1}-x^{*}\right\rangle \\
& +\left(1-\alpha_{n} \tau\right)\left\|y_{n}-x^{*}\right\|\left\|x_{n+1}-x^{*}\right\| \\
\leq & \alpha_{n} \gamma \beta\left\|x_{n}-x^{*}\right\|\left\|x_{n+1}-x^{*}\right\| \\
& +\alpha_{n}\left\langle\gamma f\left(x^{*}\right)-\mu B x^{*}, x_{n+1}-x^{*}\right\rangle \\
& +\left(1-\alpha_{n} \tau\right)\left\|x_{n}-x^{*}\right\|\left\|x_{n+1}-x^{*}\right\| \\
\leq & \left(1-\alpha_{n}(\tau-\gamma \beta)\right)\left\|x_{n}-x^{*}\right\|\left\|x_{n+1}-x^{*}\right\| \\
& +\alpha_{n}\left\langle\gamma f\left(x^{*}\right)-\mu B x^{*}, x_{n+1}-x^{*}\right\rangle \\
\leq & \alpha_{n} \gamma \beta\left\|x_{n}-x^{*}\right\|\left\|x_{n+1}-x^{*}\right\| \\
& +\alpha_{n}\left\langle\gamma f\left(x^{*}\right)-\mu B x^{*}, x_{n+1}-x^{*}\right\rangle \\
& +\left(1-\alpha_{n} \tau\right)\left\|x_{n}-x^{*}\right\|\left\|x_{n+1}-x^{*}\right\| \\
\leq & \frac{\left(1-\alpha_{n}(\tau-\gamma \beta)\right)}{2}\left(\left\|x_{n}-x^{*}\right\|^{2}+\left\|x_{n+1}-x^{*}\right\|^{2}\right) \\
& +\alpha_{n}\left\langle\gamma f\left(x^{*}\right)-\mu B x^{*}, x_{n+1}-x^{*}\right\rangle \\
& +(\tau)
\end{aligned}
$$

which hence implies that

$$
\begin{aligned}
&\left\|x_{n+1}-x\right\|^{2} \\
& \leq \frac{\left(1-\alpha_{n}(\tau-\gamma \beta)\right)}{\left(1+\alpha_{n}(\tau-\gamma \beta)\right)}\left\|x_{n}-x^{*}\right\|^{2} \\
&+\frac{2 \alpha_{n}}{1+\alpha_{n}(\tau-\gamma \beta)}\left\langle\gamma f\left(x^{*}\right)-\mu B x^{*}, x_{n+1}-x^{*}\right\rangle \\
& \leq\left(1-\alpha_{n}(\tau-\gamma \beta)\right)\left\|x_{n}-x^{*}\right\|^{2} \\
&+\frac{2 \alpha_{n}}{1+\alpha_{n}(\tau-\gamma \beta)}\left\langle\gamma f\left(x^{*}\right)-\mu B x^{*}, x_{n+1}-x^{*}\right\rangle \\
& \leq\left(1-a_{n}\right)\left\|x_{n}-x^{*}\right\|^{2}+\alpha_{n} b_{n},
\end{aligned}
$$

where

$$
\begin{gathered}
a_{n}=\alpha_{n}(\tau-\gamma \beta), \\
b_{n}=\frac{2}{1+\alpha_{n}(\tau-\gamma \beta)}\left\langle\gamma f\left(x^{*}\right)-\mu B x^{*}, x_{n+1}-x^{*}\right\rangle .
\end{gathered}
$$

It is easily seen that $\sum_{n=0}^{\infty} a_{n}=\infty$ and $\limsup _{n \rightarrow \infty} b_{n} \leq 0$ (due to (a), (b), (c), and (72)). According to Lemma 13, we can conclude that $x_{n} \rightarrow x^{*}$. This completes the proof.
Corollary 15. Let $H_{1}$ and $H_{2}$ be two real Hilbert spaces and let $\mathrm{C} \subseteq \mathrm{H}_{1}$ and $\mathrm{Q} \subseteq \mathrm{H}_{2}$ be nonempty closed subsets. Let A : $H_{1} \rightarrow H_{2}$ be a bounded linear operator. Assume that $F_{1}: C \times C \rightarrow \mathbb{R}$ and $F_{2}: Q \times Q \rightarrow \mathbb{R}$ are the bifunctions satisfying Assumption 7 and $F_{2}$ is upper semicontinuous in the first argument. Let the sequences $\left\{u_{n}\right\}$ and $\left\{x_{n}\right\}$ be generated by

$$
\begin{gathered}
u_{n}=T_{r_{n}}^{F_{1}}\left(x_{n}+\delta A^{*}\left(T_{r_{n}}^{F_{2}}-I\right) A x_{n}\right), \\
x_{n+1}=\alpha_{n} \gamma f\left(x_{n}\right)+\left(I-\mu \alpha_{n} B\right) \frac{1}{s_{n}} \int_{0}^{s_{n}} T(s) u_{n} d s .
\end{gathered}
$$

Suppose that the sequence $\left\{\alpha_{n}\right\}$ satisfies the following conditions: (a), (b), and (c) (the same in Theorem 14). Then the sequences $\left\{u_{n}\right\}$ and $\left\{x_{n}\right\}$ converge strongly to $x^{*} \in \Gamma=$ $F_{i x}(S) \bigcap \Omega$, where $x^{*}=P_{\Gamma}(I-\mu B+\gamma f) x^{*}$, which is the unique solution of the variational inequality (31).

If $B$ is a strongly positive bounded linear self-adjoint operator on $H_{1}$ with constant $\bar{\gamma}>0$ such that $0<\gamma<\bar{\gamma} / \alpha<$ $\gamma+1 / \alpha$, we can easily obtain the following corollary.

Corollary 16. Let $H_{1}$ and $H_{2}$ be two real Hilbert spaces and let $C \subseteq H_{1}$ and $Q \subseteq H_{2}$ be nonempty closed subsets. Let A : $H_{1} \rightarrow H_{2}$ be a bounded linear operator. Assume that $F_{1}: C \times C \rightarrow \mathbb{R}$ and $F_{2}: Q \times Q \rightarrow \mathbb{R}$ are the bifunctions satisfying Assumption 7 and $F_{2}$ is upper semicontinuous in the first argument. Let the sequences $\left\{u_{n}\right\}$ and $\left\{x_{n}\right\}$ be generated by

$$
\begin{gathered}
u_{n}=T_{r_{n}}^{F_{1}}\left(x_{n}+\delta A^{*}\left(T_{r_{n}}^{F_{2}}-I\right) A x_{n}\right), \\
x_{n+1}=\alpha_{n} \gamma f\left(x_{n}\right)+\left(I-\alpha_{n} B\right) \frac{1}{s_{n}} \int_{0}^{s_{n}} T(s) u_{n} d s .
\end{gathered}
$$

Suppose that the sequence $\left\{\alpha_{n}\right\}$ satisfies the following conditions: (a), (b), and (c) (the same in Theorem 14). Then the sequences $\left\{u_{n}\right\}$ and $\left\{x_{n}\right\}$ converge strongly to $x^{*} \in \Gamma=$ $F_{i x}(S) \bigcap \Omega$, where $x^{*}=P_{\Gamma}(I-\mu B+\gamma f) x^{*}$, which is the unique solution of the variational inequality

$$
\left\langle(\gamma f-B) x^{*}, \bar{x}-x^{*}\right\rangle \leq 0, \quad \forall \bar{x} \in \Gamma .
$$

\section{Application}

In this section, we introduce an example of numerical test to illustrate the algorithm given in Corollary 16.

Example 17. Let $C, Q=[0,+\infty) \in H_{1}=H_{2}=R$, the set of all real numbers, with the inner product defined by $\langle x, y\rangle=$ $x y, \forall x, y \in R$, and induced usual norm $\|\cdot\|$. Assume that $F_{1}: C \times C \rightarrow R$ and $F_{2}: Q \times Q \rightarrow R$ are defined by $F_{1}(x, y)=(x-4)(y-x), \forall x, y \in C$, and $F_{2}(u, v)=(u+$ 2) $(v-u), \forall u, v \in Q$. It is easy to claim that the bifunctions $F_{1}$ and $F_{2}$ satisfy Assumption 7 and the bifunction $F_{2}$ is upper semicontinuous. Next, we can find the formula of $T_{r_{n}}^{F_{1}} x$. From 


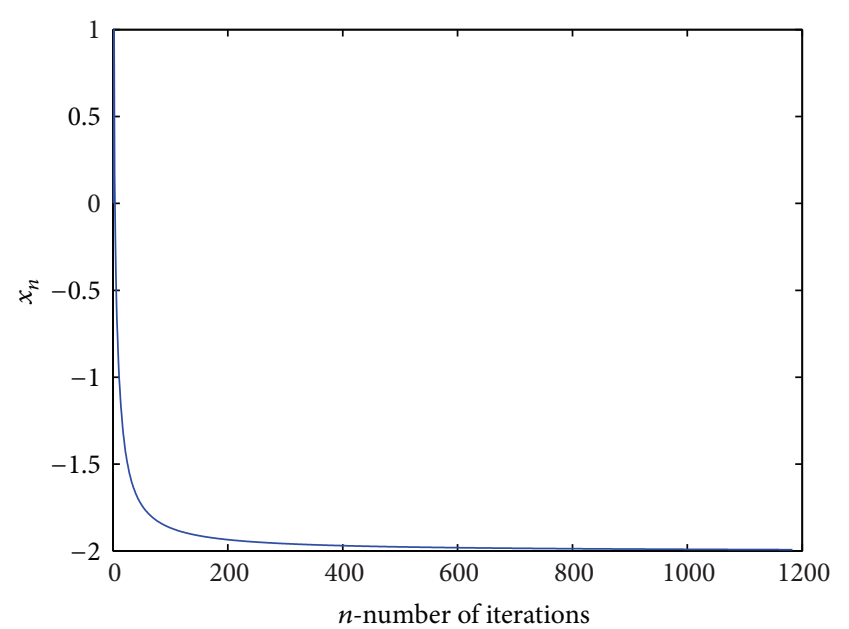

FIGURE 1: Convergence of iterative sequence $\left\{x_{n}\right\}$.

Lemma 8, we can claim that $T_{r_{n}}^{F_{1}} x$ is single-valued; for any $y \epsilon$ C, $r>0$,

$$
\begin{aligned}
& F_{1}(x, y)+\frac{1}{r}\langle x-z, y-x\rangle \geq 0 \\
& \quad \Longleftrightarrow-(r+1) x^{2}+(r x+x-4 r-z) y+(4 r+z) x \geq 0 .
\end{aligned}
$$

Letting $H(y)=-(r+1) x^{2}+(r x+x-4 r-z) y+(4 r+z) x$, we obtain that

$$
z=(r+1) x-4 r
$$

and so

$$
T_{r_{n}}^{F_{1}} x=\left(r_{n}+1\right) x-4 r_{n} .
$$

Similarly, we can also obtain that

$$
T_{r_{n}}^{F_{2}} u=\left(r_{n}+1\right) u+2 r_{n} .
$$

For all $x \in R$, we can define mapping $f(x)=(1 / 4) x$, $A(x)=-(1 / 2) x, B(x)=2 x$, and the nonexpansive mapping $T$ is satisfied as $T x=x \forall x \in C$. It is claimed that the mapping $f$ is contraction with constant $\beta=1 / 2, A$ is a bounded linear operator on $R$ with adjoint operator $A^{*}$ and $\|A\|=\left\|A^{*}\right\|=1 / 2$, and $B$ is a strongly positive bounded linear self-adjoint operator with constant $\bar{\gamma}=1$ on $R$. On the other hand, we can take $\gamma=2$ which satisfies $0<\gamma<\bar{\gamma} / \alpha<$ $\gamma+1 / \alpha$. Hence, it is easy to observe that $F_{i x}(T)=(0, \infty)$, $\mathrm{EP}\left(F_{1}\right)=\{4\}$, and $\operatorname{EP}\left(F_{2}\right)=\{-2\}$. Furthermore, we can obtain that $\Omega=\left\{p \in \mathrm{EP}\left(F_{1}\right): A p \in \operatorname{EP}\left(F_{2}\right)\right\}=\{-2\}$. Consequently, $\Gamma=F_{i x}(S) \bigcap \Omega=\{-2\}$. Therefore, all the assumptions in Corollary 16 are satisfied. We can obtain the following numerical algorithm:

$$
\begin{gathered}
u_{n}=T_{r_{n}}^{F_{1}}\left(x_{n}+\frac{1}{8} A^{*}\left(T_{r_{n}}^{F_{2}}-I\right) A x_{n}\right), \\
x_{n+1}=\frac{2}{n+1}\left(\frac{1}{4} x_{n}\right)+\left(1-\frac{2}{n+1}\right) u_{n},
\end{gathered}
$$

where $\alpha_{n}=1 /(n+1)$ and $r_{n}=1$. Then, by Corollary 16, the sequence $\left\{x_{n}\right\}$ converges to a solution of Example 17. For a number $\varepsilon=10^{-5}$, using the MATLAB, we generated a sequence $\left\{x_{n}\right\}$ as in Figure 1 .

\section{Conflict of Interests}

The authors declare that there is no conflict of interests regarding the publication of this paper.

\section{References}

[1] E. Blum and W. Oettli, "From optimization and variational inequalities to equilibrium problems," The Mathematics Student, vol. 63, no. 1-4, pp. 123-145, 1994.

[2] P. L. Combettes and S. A. Hirstoaga, "Equilibrium programming in Hilbert spaces," Journal of Nonlinear and Convex Analysis, vol. 6, no. 1, pp. 117-136, 2005.

[3] Y. Censor and A. Segal, "The split common fixed point problem for directed operators," Journal of Convex Analysis, vol. 16, no. 2, pp. 587-600, 2009.

[4] P. Kumam and C. Jaiboon, "A new hybrid iterative method for mixed equilibrium problems and variational inequality problem for relaxed cocoercive mappings with application to optimization problems," Nonlinear Analysis: Hybrid Systems, vol. 3, no. 4, pp. 510-530, 2009.

[5] Q. Liu, W. Zeng, and N. Huang, "An iterative method for generalized equilibrium problems, fixed point problems and variational inequality problems," Fixed Point Theory and Applications, vol. 2009, Article ID 531308, 20 pages, 2009.

[6] K. R. Kazmi and F. A. Khan, "Existence and iterative approximation of solutions of generalized mixed equilibrium problems," Computers \& Mathematics with Applications, vol. 56, no. 5, pp. 1314-1321, 2008.

[7] P. Kumam, N. Petrot, and R. Wangkeeree, "A general system of variational inequality problems and mixed equilibrium problems," Applied Mathematics and Mechanics, vol. 29, no. 5, pp. 571-581, 2008.

[8] Y. Yao, Y.-C. Liou, and J.-C. Yao, "Convergence theorem for equilibrium problems and fixed point problems of infinite family of nonexpansive mappings," Fixed Point Theory and Applications, vol. 2007, Article ID 64363, 12 pages, 2007.

[9] A. Moudafi, "Split monotone variational inclusions," Journal of Optimization Theory and Applications, vol. 150, no. 2, pp. 275283, 2011.

[10] M. Tian, "A general iterative algorithm for nonexpansive mappings in Hilbert spaces," Nonlinear Analysis, vol. 73, no. 3, pp. 689-694, 2010.

[11] L. C. Ceng, Q. H. Ansari, and J. C. Yao, "Some iterative methods for finding fixed points and for solving constrained convex minimization problems," Nonlinear Analysis: Theory, Methods \& Applications, vol. 74, no. 16, pp. 5286-5302, 2011.

[12] S. Plubtieng and R. Punpaeng, "Fixed-point solutions of variational inequalities for nonexpansive semigroups in Hilbert spaces," Mathematical and Computer Modelling, vol. 48, no. 12, pp. 279-286, 2008.

[13] K. R. Kazmi and S. H. Rizvi, "Implicit iterative method for approximating a common solution of split equilibrium problem and fixed point problem for a nonexpansive semigroup," Arab Journal of Mathematical Sciences, vol. 20, no. 1, pp. 57-75, 2014. 
[14] K. Goebel and W. A. Kink, Topics on Metric Fixed Point Theory, Gambridge University Press, Gambridge, UK, 1990.

[15] T. Shimizu and W. Takahashi, "Strong convergence to common fixed points of families of nonexpansive mappings," Journal of Mathematical Analysis and Applications, vol. 211, no. 1, pp. 7183, 1997.

[16] V. Colao, G. L. Acedo, and G. Marino, "An implicit method for finding common solutions of variational inequalities and systems of equilibrium problems and fixed points of infinite family of nonexpansive mappings," Nonlinear Analysis: Theory, Methods \& Applications, vol. 71, no. 7-8, pp. 2708-2715, 2009.

[17] I. Yamada, "The hybrid steepest descent method for the variational inequality problem over the intersection of fixed point sets of nonexpansive mappings," Studies in Computational Mathematics, vol. 8, pp. 473-504, 2001.

[18] H. K. Xu, "Iterative algorithms for nonlinear operators," Journal of the London Mathematical Society, vol. 66, no. 1, pp. 240-256, 2002. 


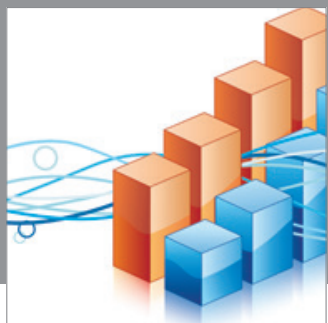

Advances in

Operations Research

mansans

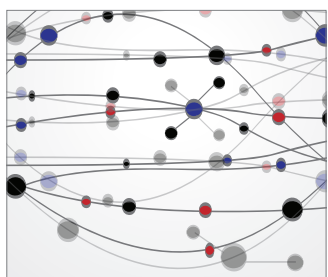

The Scientific World Journal
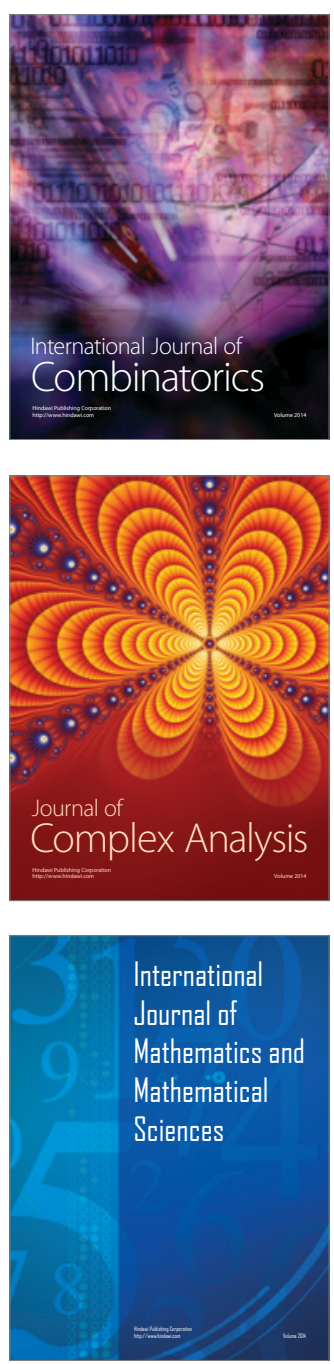
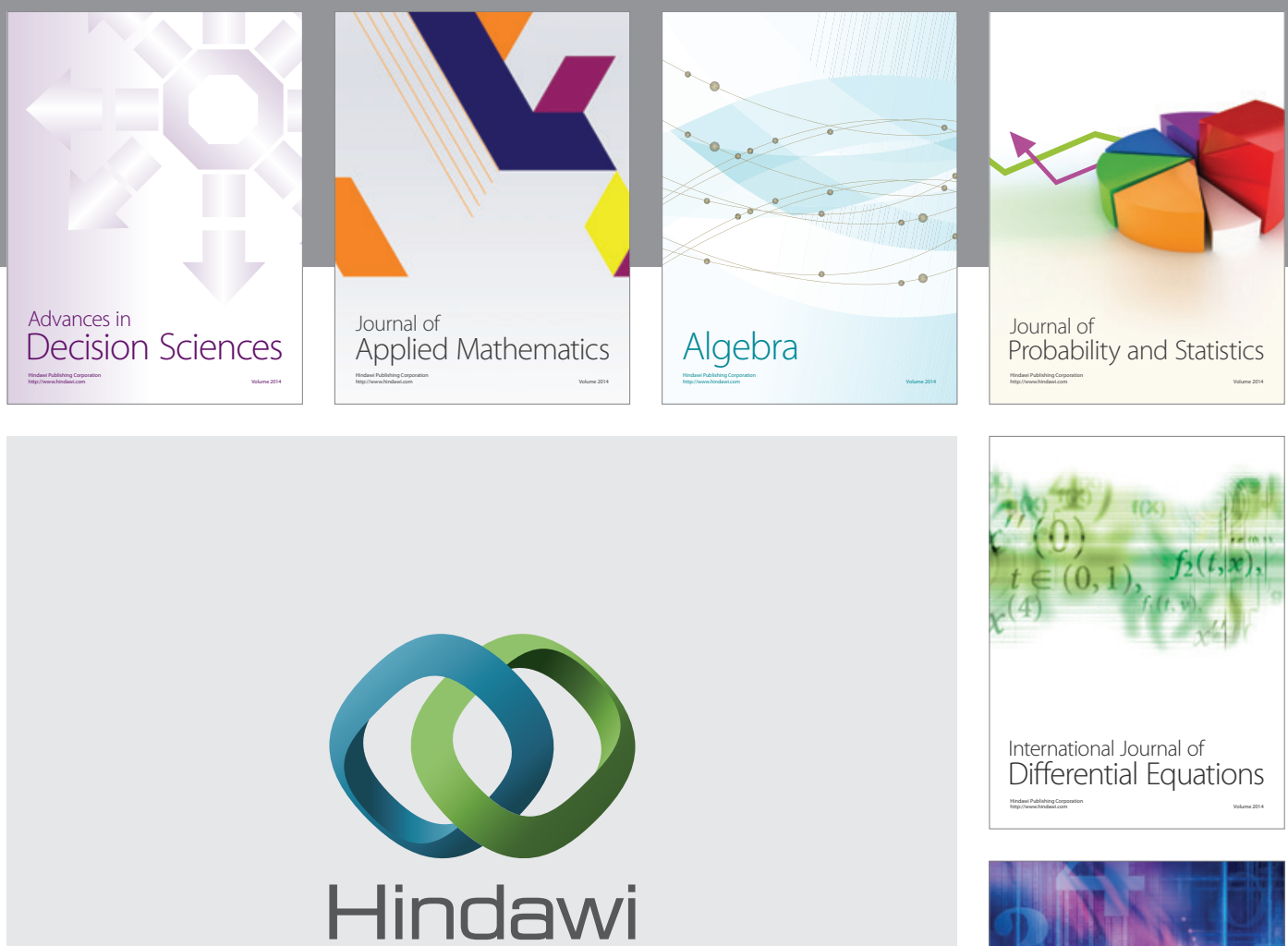

Submit your manuscripts at http://www.hindawi.com
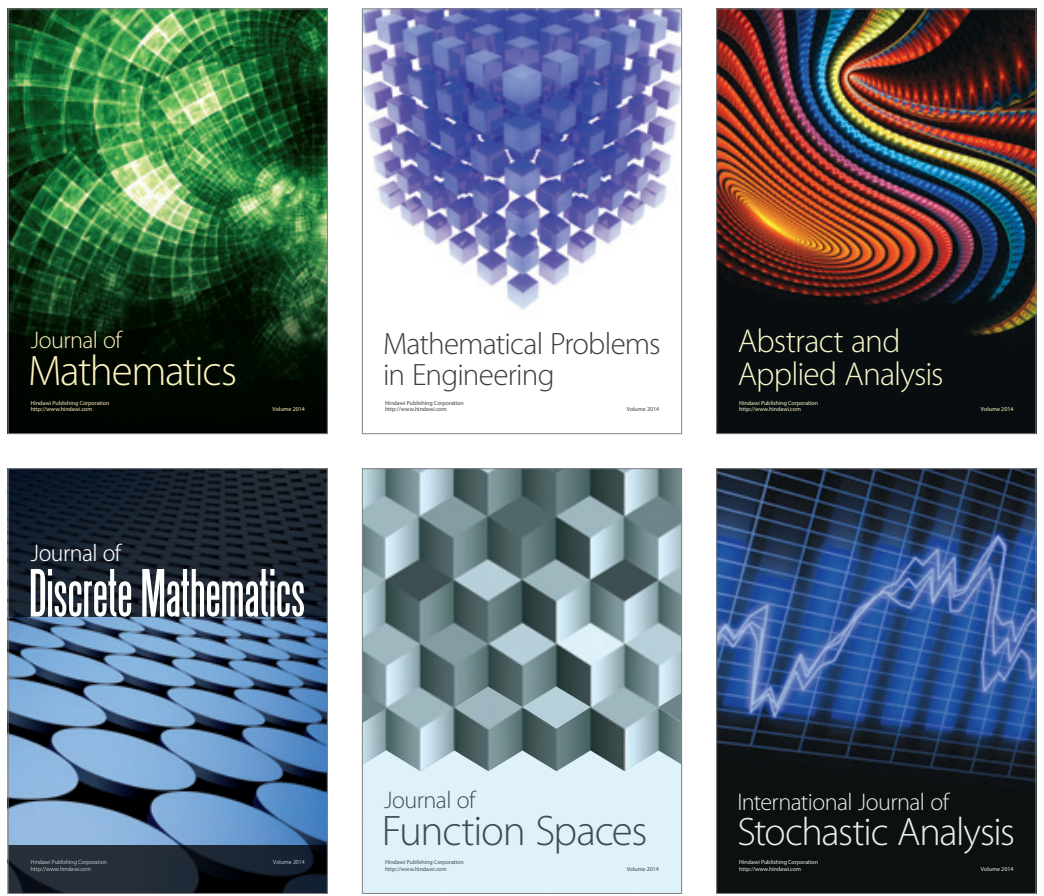

Journal of

Function Spaces

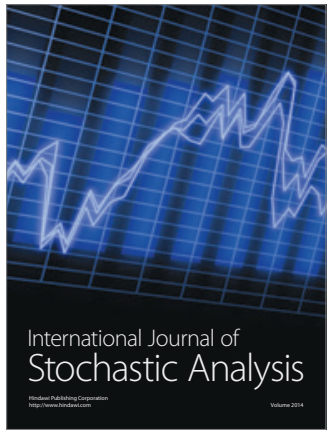

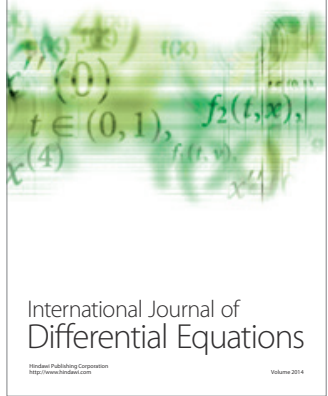
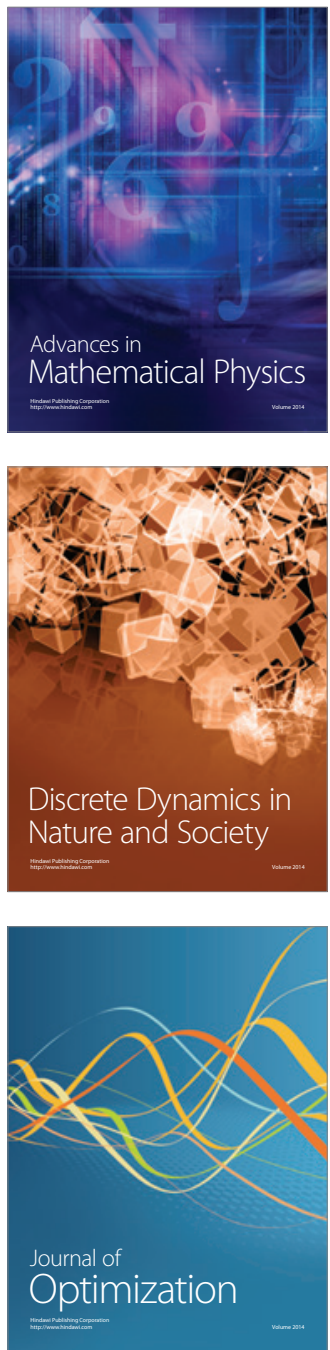